\title{
Plasma sublimation for the treatment of xanthelasma palpebrarum
}

\author{
Silvestrs Rubins ${ }^{1,2}$, Ingrida Ritina3 ${ }^{3}$ Jeannette Jakus4, Andris Rubins ${ }^{1,2} \bowtie$ \\ ${ }^{1}$ Department of Dermatovenerology, Faculty of Medicine, University of Latvia, Riga, Latvia. ${ }^{2}$ Latvian Dermatology Institute, Riga, Latvia. ${ }^{3}$ D.A.P. Clinic, \\ Riga, Latvia. ${ }^{4}$ Departament of Dermatology, SUNY Downstate Health Sciences University, New York, NY, USA.
}

\begin{abstract}
Introduction: Xanthelasma palpebrarum (XP) is a common xanthomatous lesion of the eyelid and periorbital skin. Several methods of treatment have been reported in the literature, each having its own indications and risks. We present a new treatment method for removing XP using a plasma exeresis device (Plexr ${ }^{\circledR}, \mathrm{GMV}$, Italy).

Methods: Fifteen patients with a total of 27 treated XPs were assessed and clinically identified by the treating dermatologist. Patients were photographed and assessed by a dermatologist prior to and immediately after treatment. A patient survey was conducted 12 months after the procedure, which assessed the outcome of the procedure (redness, pigmentation disorders, and scars). Results: After just a single treatment session using a plasma sublimation, all 27 XPs showed complete clearance. There were no reports of scars, pigmentary alteration, or recurrence of lesions up to 12 months.

Conclusions: We present plasma sublimation as a new method for the treatment of XP. During the procedure, the method allows to control the depth of tissue destruction and the presence of xanthoma tissue, and to minimize pain and trauma, making it particularly ideal for treating areas around the eye.
\end{abstract}

Keywords: plasma sublimation, xanthelasma palpebrarum, aesthetic medicine

Received: 1 December 2019| Returned for modification: 11 March 2020 | Accepted: 23 March 2020

\section{Introduction}

Xanthelasma palpebrarum (XP) is a common localized condition of the eyelid and periorbital skin due to the accumulation of lipidrich macrophages, known as foam cells.

Clinically, xanthelasmas appear as flat or slightly elevated yellowish-tan lesions in middle-aged or elderly patients. XP can present as soft, semisolid, or calcified papules, plaques, or nodules. The inner canthus of the upper lid is the area most frequently involved (Fig. 1); however, the lower lid or periorbital skin can also be affected (1). Lesions typically occur symmetrically. XPs are progressive and permanent, and they can only be resolved with treatment. Approximately $50 \%$ of patients have underlying hyperlipidemia, with type IIa hyperlipidemia most commonly reported (2). According to data published by Pe'er, most patients with XP are normolipidemic (3). Based on this information, we believe that lipid levels were not significant in our study.

Histologically, XP is composed of collections of lipid-laden histiocytes in the superficial dermis, mainly surrounding blood vessels and adnexae (4).

Although generally benign, XPs may cause significant cosmetic and functional disfigurement. Several methods of treatment have been reported in the literature, including surgery, cryotherapy, electrodessication, dermabrasion, trichloroacetic acid (TCA), lasers, and radiofrequency (RF). Each has its own respective indications and risks. For example, definitive surgery may sometimes involve general anesthesia and may lead to scarring and functional impairment with larger lesions (5). Lasers can have good results, but several treatment sessions are often required, and this can be costly (6). With other methods such as cryotherapy, depth of penetration can be difficult to judge, leading to uncertain efficacy and adverse events (7). It is therefore important for the treating physician to be aware of and choose the most appropriate method when considering treatments for XP. For both patients and physicians, the ideal treatment for XP should be safe, comfortable, and affordable, and provide total clearance with no recurrence while minimizing unwanted side effects. We present a new method for the treatment of XP using plasma sublimation.

\section{Methods}

A retrospective review was conducted of all patients with XP treated with plasma sublimation from November 2013 through November 2017 at the Latvian Dermatology Institute in Riga, Latvia. This study was approved by the Ethics Committee of the Latvian Institute of Cardiology for Clinical and Physiological Research, and Drug and Pharmaceutical Product Clinical Investigation.

Fifteen patients with a total of 27 XPs treated with plasma sublimation were identified. All XP lesions were assessed by a dermatologist and clinically confirmed on dermoscopy. Treatment sites were prepped using an antiseptic cleanser (Octenisept ${ }^{\circledR}$ ) followed by either local anesthesia ( $2 \%$ lidocaine solution) or a topical eutectic mixture of lidocaine (EMLA) under occlusion for 40 minutes. Eye protection is not indicated during use of the Plexr ${ }^{\circledR}$, and patients were asked to keep their eyes closed. Treatments were performed using the Plexr ${ }^{\circledR}$ (GMV, Italy) plasma-generating device (output frequency up to $70 \mathrm{kHz}$ and maximum output power $<2$ W) on the high power setting and "spray" technique, evenly treating the entire surface in a layered approach until complete clinical clearance was achieved. The carbonized deposits were removed from time to time and the procedure was repeated various times until they disappeared. The number of passes varied depending on the thickness of each lesion. A smoke aspirator was used to remove smoke from the field. Photographs were taken of all lesions prior to and immediately following treatment (Figs. 1-2). After the procedure, the treated areas were cooled and cleansed again using 
the same antiseptic cleanser. Patients were sent home with instructions to cleanse the treated areas with an antiseptic cleanser without alcohol (Octenisept ${ }^{\circledR}$ ) and with water twice a day for four days and to return to the clinic for post-procedure assessments and photographs once all crusting had subsided (average 7 to 14 days; Fig. 3). A patient survey was conducted 12 months after the procedure, which assessed the outcome of the procedure (redness, pigmentation disorders, and scars). Several patients sent photos.

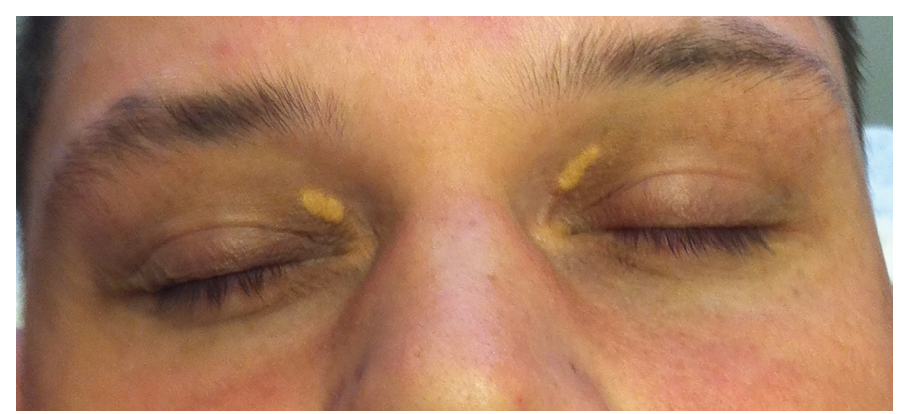

Figure 1 | Xanthelasma palpebrarum before treatment.

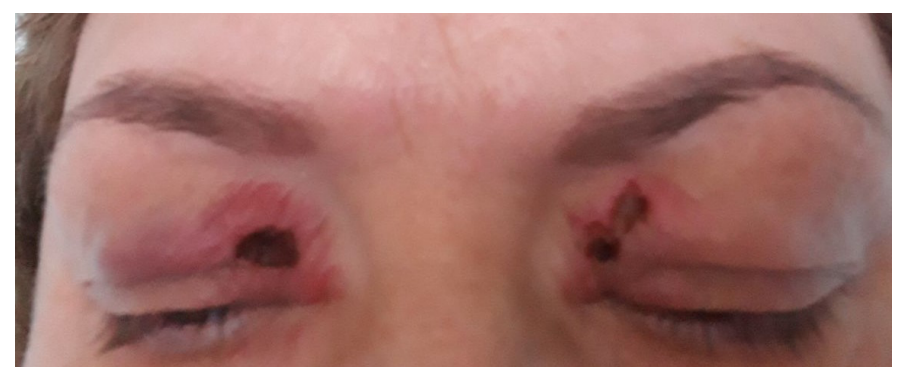

Figure 2 | Immediately after treatment with plasma sublimation.

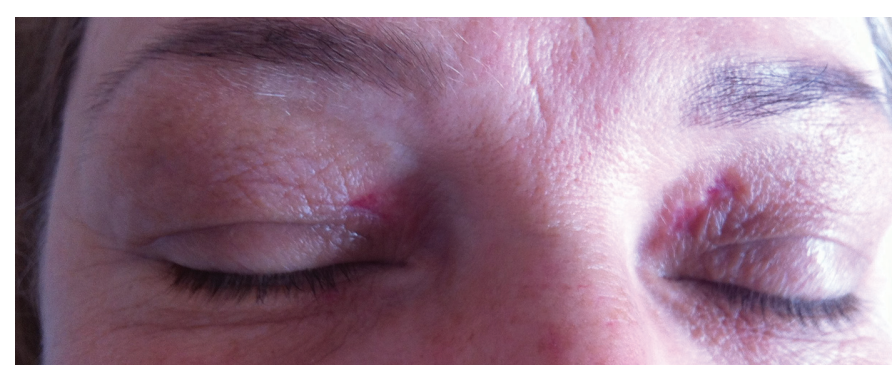

Figure 3 | Fourteen days after treatment.

\section{Results}

Fifteen patients with a total of 27 XP lesions were treated by plasma sublimation at our institution between November 2013 and November 2017.

Twelve patients had bilateral lesions of XP. Three had only one lesion on the upper eyelid. All 27 XP lesions resolved completely with just one treatment with plasma sublimation. Patients tolerated the procedure well with no intraoperative complications or pain. No serious adverse events were experienced. All patients developed a brown crust at the site of treatment at the time of the procedure, lasting on average for 7 to 14 days. Following resolution of the crust, patients experienced full restoration of their normal skin texture over a period of 1 month post-procedure, with some experiencing mild residual pale erythema over this time. No scars or pigmentary changes were reported. No recurrence of lesions was detected during the follow-up period and up to 12 months post-procedure. Patients evaluated the procedure as comfortable, requiring minimal and easy aftercare. All were satisfied with the cosmetic result.

\section{Discussion}

Many patients consider XP to be an aesthetic defect, and they visit dermatologists and aesthetic medicine specialists to remove them. Due to the risky location near the eye and high recurrence rate, effective treatment of XP can be difficult. Local treatment options include surgical resection, electrodesiccation, RF, cryosurgery, chemical cauterization, and ablation with lasers $(5,7-10,12,13)$. However, each method of treatment is associated with limitations and complications, including persistent erythema, recurrence, scarring, ectropion, and pigmentary alteration. Labandeira et al., for example, share the experience of removing xanthelasma with liquid nitrogen, highlighting the simplicity of this technology and its low cost. However, this method is rarely used due to inaccuracy of depth of treatment, high risk of pigmentary alteration, and pronounced swelling of the eyelids (8). RF is a safe and effective treatment method in XP with satisfactory cosmetic results; side effects such as hyperpigmentation, hypopigmentation, and ectropion are rarely seen (9). A study by Reddy et al. has compared the efficacy of RF ablation versus TCA in the treatment of XP (10). This was a split-face trial of twenty patients with bilateral XP in which each patient was treated with RF ablation on one side and TCA application on the other side. At 4 weeks follow-up, $40 \%$ of XPs treated with RF and $15 \%$ of those treated with TCA resulted in scar formation. Forty-five percent of the lesions treated with $\mathrm{RF}$ and $30 \%$ of those treated with TCA resulted in post-procedural hyperpigmentation. The authors concluded that, although RF ablation required fewer sessions for achieving more than $75 \%$ clearance of lesions, TCA applications were associated with fewer complications comparatively.

A few recent publications examine the use of lasers for the treatment of XP. In the largest review to date, Nguyen et al. found a wide range of lasers being used, with $10,600 \mathrm{~nm} \mathrm{CO} 2$ being the most frequently reported (11). They conclude that, overall, lasers provide moderate to excellent clearance rates with minimal side effects. Efficacy, the number of treatment sessions, risk of dyspigmentation, scarring, and length of down time were highly variable, mostly depending on the type of laser being used. The costs of treatment to the physician and patient were not assessed.

Recurrence of XP is a common problem (12). In order to prevent a relapse, it is essential to completely destroy all associated xanthoma cells, which can be a challenge when using most existing modalities because depth of treatment penetration can be difficult to assess and control. This is particularly an issue when xanthomas infiltrate eyelid muscle, a rare but noted occurrence. $\mathrm{CO}_{2}$ laser devices typically have a controlled depth of penetration, although thermal damage to surrounding deep tissue can occur, leading to pigmentary alteration and the potential for scarring (13).

For both patients and physicians, the ideal treatment for XP should be safe, comfortable, and affordable, and should provide total clearance with no recurrence while minimizing unwanted side effects such as scarring and pigmentary alteration. Given the limitations of currently available modalities in the treatment of $\mathrm{XP}$, it is clear that there remains an unmet need.

To address this, we present plasma sublimation as a novel method for the successful treatment of XP. Plasma sublimation is an electrosurgical, non-ablative technique in which radio frequency energy generates plasma from atmospheric gas, creating a cocktail of intermittently mobile ions, electrons, and chemical compounds. The focused micro-plasma beam sublimates epidermal targeted tissue: a change in tissue from solid to gaseous phase. 
The ionic flow does not spread to the deeper tissue, the basal lamina is preserved, and tissue is targeted via evaporation, thus preventing heat transfer and subsequent damage to surrounding tissue, leading to very controlled and precise tissue destruction (14).

The advantages of this procedure include its excellent efficacy, minimal downtime, and high safety profile. Plasma sublimation devices are small, portable, and easy to use, making them ideal for in-office use. The cost of these devices varies widely, although they are generally affordable and less costly than most laser equipment. The safety and efficacy of plasma sublimation has been well demonstrated in non-surgical blepharoplasty procedures and for removal of other benign skin lesions $(14,15)$. The risk of damage to the eye is minimal, and protective eyewear is not indicated for either the provider or patient, making it a safe option for use around the eye.

In our retrospective analysis of 15 patients with 27 XPs treated by plasma sublimation, we found 100\% clearance of lesions with just a single treatment. All the wounds re-epithelialized within 1 to 2 weeks of treatment, resulting in minimal downtime for patients. Some patients experienced residual pale pink erythema

\section{References}

1. Baykal C, Polat Ekinci A, Yazganoglu KD, Buyukbabani N. The clinical spectrum of xanthomatous lesions of the eyelids. Int J Dermatol. 2017;56:981-92.

2. Sotiriadis D, Patsatsi A. European handbook of dermatological treatments. 3 rd ed. Heidelberg: Springer; 2015. Chapter 107, Xanthomas; p. 1063-9.

3. Pe'er J. Pathology of eyelid tumors. Indian J Ophthalmol. 2016;64:177-90.

4. Bergman R. The pathogenesis and clinical significance of xanthelasma palpebrarum. J Am Acad Dermatol. 1994;30:236-42.

5. Mendelson BC, Masson JK. Xanthelasma: follow-up on results after surgical excision. Plast Reconstr Surg. 1976;58:535-8.

6. Sebaratnam DF, Lim AC, Lowe PM, Goodman GJ, Bekhor P, Richards S. Lasers and laser-like devices: part two. Australas J Dermatol. 2014;55:1-14.

7. Labandeira J, Vázguez-Osorio I, Figueroa-Silva 0, Pereiro MJ, Toribio J. Tolerability and effectiveness of liquid nitrogen spray cryotherapy with very short freeze time in the treatment of xantelasma palpebrarum. Dermatol Ther. 2015;28:346-50.

8. Marini LG, Krunic AL. European handbook of dermatological treatments. 3rd ed. Heidelberg: Springer; 2015. Chapter 121, Laser: ablative; p. 1223-37.

9. Nair PA, Singhal R. Xanthelasma palpebrarum-a brief review. Clin Cosmet Investig Dermatol. 2017;11:1-5. over the 1st month; however, there were no reports or evidence of scarring, dyspigmentation, or recurrence up to 12 months postprocedure. The treating physicians reported simplicity and safety of manipulation with complete control of volume and depth of tissue destruction. The patients also expressed complete satisfaction with the cosmetic outcomes of their treatments, with no reports of pain or discomfort during or after the procedure. Even though this study is limited by our small sample size, retrospective design, and lack of a control group, we conclude that plasma sublimation can be considered a valuable modality for the treatment of XP. Although larger controlled studies are needed, this treatment appears to provide comparable if not superior results compared to those reported with currently existing modalities.

\section{Conclusions}

Our results support the use of plasma sublimation in the treatment of XP. This device provides positive results with maximum control and easy handling, making it ideal for use around the eye.

10. Reddy PK, Kunneth ST, Lakshminarayana SP, Yallappa SM, Chandrashekara R, Nanjundaswamy SK. Comparative study to evaluate the efficacy of radiofrequency ablation versus trichloroacetic acid in the treatment of xanthelasma palpebrarum. J Cutan Aesthet Surg. 2016;9:236-40.

11. Nguyen AH, Vaudreuol AM, Huerter CJ. Systematic review of laser therapy in xanthelasma palpebrarum. Int J Dermatol. 2017;56:e47-e55.

12. Rohrich RJ, Janis JE, Pownell PH. Xanthelasma palpebrarum: a review and current management principles. Plast Reconstr Surg. 2002;110:1310-4.

13. Raulin C, Schoenermark MP, Werner S, Greve B. Xanthelasma palpebrarum: treatment with the ultrapulsed CO2 laser. Lasers Surg Med. 1999;24:122-7.

14. Scarano A, Carinci F, Sinjari B, Artese L, Fippi G, Brunelli G, et al. Skin lesions induced from the radiosurgical unit and voltaic arc dermoabrasion: a rabbit model. Eur J Inflamm. 2011;9:89-94.

15. Ritina IF, Rubins SA, Rubins AY. Experience of removing benign neoplasms of skin in cervicofacial area using the method of plasma ablation. Vestn Dermatol Venerol. 2017;93:44-52. 